\title{
Conjunctival Microangiopathy in a Patient with Neovascular Glaucoma
}

\author{
Anmar M. Abdul-Rahman ${ }^{a}$ Anthony Molteno ${ }^{b}$ \\ ${ }^{a}$ Department of Ophthalmology, Counties Manukau DHB, Auckland, and ${ }^{b}$ Ophthalmology \\ Section, Dunedin School of Medicine, University of Otago, Dunedin, New Zealand
}

\section{Key Words}

Conjunctival microangiopathy · Diabetes - Neovascular glaucoma $\cdot$ Anterior segment angiography $\cdot$ Glaucoma drainage implant

\begin{abstract}
Purpose: To report a case of conjunctival microangiopathy secondary to diabetes presenting as a red eye 5 years after glaucoma implant surgery for neovascular glaucoma. Materials and Methods: A 52-year-old Tongan male with a past history of neovascular glaucoma secondary to proliferative diabetic retinopathy underwent slitlamp examination and color photography of the anterior and posterior segment, in addition to anterior segment fluorescein angiography. Results: The conjunctival venules perilimbally demonstrated tortuosity and dilation (saccular, fusiform and tricornuate). Capillaries were tortuous, with sparse microaneurysm formation, and arterioles were attenuated. Anterior segment fluorescein angiography showed generalized iris ischemia, extensive perilimbal microvascular degenerative changes and leakage of fluorescein into the anterior chamber. We are unaware of previous reports in the literature on conjunctival microangiopathy secondary to diabetes presenting as a red eye. Conclusions: We hypothesized that the clinical features in our case may have been modified by a combination of postoperative wound healing and degenerative vascular remodeling, in addition to microvascular alterations secondary to both diabetes and consequent ocular ischemia, which may have played a role.

(c) 2015 S. Karger AG, Basel
\end{abstract}

\section{Introduction}

Conjunctival microangiopathy are nonspecific, morphologic and hemodynamic changes associated with senility, degenerative vascular disease, rheumatic fever and sickle cell crisis and in normal adults after the third decade [1]. 
Abdul-Rahman and Molteno: Conjunctival Microangiopathy in a Patient with Neovascular Glaucoma

They mainly involve the capillary and postcapillary venules of the conjunctival microvascular network, and alterations include increased capillary tortuosity, angularity, elongation and microaneurysm formation, as well as venular distention, tortuosity and focal dilation (saccular, fusiform or tricornuate). Arterioles demonstrated generalized narrowing with focal constrictions and loss of transparency [2].

We describe a case where the severity of the conjunctival microangiopathy resulted in a painless unilateral red eye as the primary complaint, 5 years after successful control of underlying proliferative diabetic retinopathy and neovascular glaucoma (NVG). We are unaware of previous reports in the literature on conjunctival microangiopathy secondary to diabetes presenting as a red eye.

\section{Case Report}

A 52-year-old Tongan male presented with a unilateral painless red right eye, with 3 months of gradual onset. His past medical history included type II diabetes diagnosed 16 years ago, with poor systemic control, hypertension and hyperlipidemia.

His past ocular history included bilateral pseudophakia and bilateral panretinal photocoagulation for proliferative diabetic retinopathy 5 years prior to the current presentation. This was complicated by progression to NVG in the right eye after 2 months, with a presenting intraocular pressure (IOP) of $58 \mathrm{~mm} \mathrm{Hg}$. Gonioscopy revealed peripheral anterior synechiae involving three quadrants. IOP was stabilized with a superior temporally placed 230$\mathrm{mm}^{2}$ M3 Molteno implant, intravitreal bevacizumab and further panretinal photocoagulation. The patient remains off topical treatment.

On examination at the current presentation, his visual acuity was 6/9 OD and 6/12 OS, and IOP was $18 \mathrm{~mm} \mathrm{Hg} \mathrm{OU.} \mathrm{Slitlamp} \mathrm{examination} \mathrm{of} \mathrm{the} \mathrm{anterior} \mathrm{segment} \mathrm{of} \mathrm{the} \mathrm{right} \mathrm{eye}$ revealed venular dilation, beading, focal dilation (saccular, fusiform and tricornuate) and tortuosity. Capillaries demonstrated microaneurysm formation and tortuosity, with attenuated arterioles. The vascular changes involved the bulbar conjunctiva circumferentially, predominantly inferonasally. The changes were absent over both the bulbar conjunctiva of the bleb wall and the tarsal conjunctiva. The cornea showed inferior nasal early arborescent neovascularization with a pseudopterygium $1.2 \mathrm{~mm}$ from the temporal limbus (fig. 1).

The pupil was oval with the long axis temporally superior, and the iris demonstrated inferior sectoral atrophy involving 2 clock hours. Posterior segment examination showed a $\mathrm{C} / \mathrm{D}$ ratio of 0.6 with inferior thinning and peripheral laser scars. The retinal vasculature of the venous tree appeared normal in caliber; however, the retinal arterioles were attenuated and hyalinized (fig. 2).

Anterior segment fluorescein angiography demonstrated an absence of vascular flow in the iris, except at the pupillary margin, extensive limbal microaneurysms, partial filling of the saccular dilations and vascular tortuosity.

The inferior nasal cornea demonstrated early neovascularization. Leakage of fluorescein into the anterior chamber was noted after 1 min of fluorescein injection (fig. 3).

No changes were demonstrated in the clinical findings over a period of observation of 5 months. 
Abdul-Rahman and Molteno: Conjunctival Microangiopathy in a Patient with Neovascular Glaucoma

\section{Discussion}

Ernst Coccius [3] was the first to observe the circulation in the conjunctival vessels in 1852. The recognition of an association between systemic degenerative vascular disease and the bulbar conjunctival microcirculation lagged behind the knowledge of this association with the retinal circulation by several decades $[2,4]$.

We postulate that the conjunctival vascular changes in our case are the result of a combination of three main factors: (1) vascular remodeling secondary to postoperative conjunctival wound healing, (2) degenerative vascular changes secondary to diabetes, and (3) factors related to NVG.

Vascular Remodeling Secondary to Postoperative Conjunctival Wound Healing

The complex and dynamic process of wound healing is divided into three phases: inflammatory, proliferative and remodeling [5].

Angiogenesis occurs during the proliferative phase, signaled by the interaction of vascular endothelial growth factors (VEGFs) and their receptors. In mature vessels, angiopoietin (Ang1) binding to receptor Tie2 maintains the association of periendothelial support cells. Binding Ang2 to receptor Tie2 loosens the contact between the vascular structures, the extracellular matrix and the perivascular cells, allowing access to angiogenic factors. Other factors such as platelet-derived growth factor and transforming growth factor- $\beta$ (TGF- $\beta$ ) and their receptors are important for vascular maturation and remodeling [6].

This dynamic mechanism may have played a significant role in the development of the clinical features seen in our case, evidenced by the absence of similar findings in the contralateral eye that did not undergo a conjunctival peritomy necessary for glaucoma implant surgery.

Degenerative Vascular Changes Secondary to Diabetes

Bulbar conjunctival microvascular changes in type II diabetes include conjunctival edema, hyaline-like infiltration, hemorrhage and intravascular cellular aggregation. Morphologic vascular changes include capillary tortuosity, venous and capillary elongation, localized venous dilation and sacculation. Alterations in the arteriolar:venular ratio range from 1:3 to $1: 2$ in normal subjects to $1: 6$ in diabetics $[1,7]$, with a reduction in vessel density by $14 \%$ and an increase in mean vessel width by $5 \%$ [8].

Factors that modify the clinical picture include the grade of diabetic retinopathy, which is positively correlated with the severity of conjunctival microangiopathy [9] and the duration of diabetes, which is associated with both an overall reduction in tortuosity in macrovessels and an increased tortuosity in capillary-sized vessels [4].

The multistep process of angiogenesis is tightly controlled by a dynamic balance between positive and negative regulators. Positive regulators of angiogenesis include VEGF-A, fibroblast growth factor, angiopoietins, TGF- $\alpha$ and TGF- $\beta$, hepatocyte growth factor, connective tissue growth factor and interleukin-8. There is considerable evidence that VEGF-A is a prime regulator of angiogenesis. VEGF-A stimulates endothelial cells to degrade their basement membrane and to migrate with the concomitant release of matrix metalloproteinases and plasminogen activators and the expression of $\alpha_{v} \beta_{3}$ and $\alpha_{v} \beta_{5}$ integrins. Furthermore, VEGF-A is able to stimulate endothelial cells to proliferate, form tubes in vitro and survive [10].

Delayed onset of the conjunctival findings may be explained through diabetes-induced endothelial cell dysfunction, causing dysregulation of angiogenic factors and subsequent aberrant angiogenic responses, primarily through the increased production of reactive oxy- 
Abdul-Rahman and Molteno: Conjunctival Microangiopathy in a Patient with Neovascular Glaucoma

gen species via the activity of enzymes, such as nicotinamide adenine dinucleotide phosphate oxidase, aldehyde oxidase, xanthine oxidase and glucose oxidase, and in addition to mitochondrial uncoupling [11].

Our findings are consistent with descriptions of other investigators. The clinically evident severity of retinopathy and the duration of diabetes would have influenced the nature, delayed onset and progression of the clinical pattern.

\section{Factors Related to NVG}

NVG is a risk factor for conjunctival melt and glaucoma implant exposure (odds ratio 28.5), which suggests preexisting conjunctival microcirculatory abnormalities in this group of patients [12]. The lack of vascular flow in the iris as indicated by fluorescein angiography indicates a severe degree of ocular ischemia [13]. In addition, NVG increases the levels of VEFG and placental growth factor in the aqueous humor [14].

In our case, the patient's history of NVG, ongoing ocular ischemia and the influences of angiogenic factors in the aqueous humor, and even the absence of active proliferative changes, may have influenced the conjunctival vascular changes.

\section{Conclusion}

We hypothesize that the clinical features in our case may have been modified by a combination of postoperative wound healing and degenerative vascular remodeling, in addition to microvascular alterations secondary to both diabetes and consequent ocular ischemia, which may have played a role.

\section{Statement of Ethics}

The patient's informed consent has been obtained.

\section{Disclosure Statement}

The co-author is the inventor of the Molteno implant.

\section{References}

1 Ditzel J, Sagild U: Morphologic and hemodynamic changes in the smaller blood vessels in diabetes mellitus. 2. The degenerative and hemodynamic changes in the bulbar conjunctiva of normotensive diabetic patients. N Engl J Med 1954;250:587-594.

-2 Elliott FA, Leonberg SC Jr: Conjunctival microangiopathy. An early sign of degenerative vascular disease? Am J Med 1977;63:208-214.

3 Coccius E: Über die Ernährungsweise der Hornhaut und die Serum führenden Gefässe im menschlichen Körper. Leipzig, Müller, 1852.

4 Owen CG, Newsom RS, Rudnicka AR, et al: Diabetes and the tortuosity of vessels of the bulbar conjunctiva. Ophthalmology 2008;115:e27-e32.

5 Cordeiro MF, Chang L, Lim KS, et al: Modulating conjunctival wound healing. Eye (Lond) 2000;14:536-547.

-6 Karamysheva AF: Mechanisms of angiogenesis. Biochemistry (Mosc) 2008;73:751-762.

7 Cheung AT, Ramanujam S, Greer DA, et al: Microvascular abnormalities in the bulbar conjunctiva of patients with type 2 diabetes mellitus. Endocr Pract 2001;7:358-363. 
Abdul-Rahman and Molteno: Conjunctival Microangiopathy in a Patient with Neovascular Glaucoma

8 Owen CG, Newsom RS, Rudnicka AR, et al: Vascular response of the bulbar conjunctiva to diabetes and elevated blood pressure. Ophthalmology 2005;112:1801-1808.

-9 To WJ, Telander DG, Lloyd ME, et al: Correlation of conjunctival microangiopathy with retinopathy in type-2 diabetes mellitus (T2DM) patients. Clin Hemorheol Microcirc 2011;47:131-141.

10 Witmer AN, Vrensen GF, Van Noorden CJ, et al: Vascular endothelial growth factors and angiogenesis in eye disease. Prog Retin Eye Res 2003;22:1-29.

$\checkmark 11$ Kolluru GK, Bir SC, Kevil CG: Endothelial dysfunction and diabetes: effects on angiogenesis, vascular remodeling, and wound healing. Int J Vasc Med 2012;2012:918267.

12 Koval MS, El Sayyad FF, Bell NP, et al: Risk factors for tube shunt exposure: a matched case-control study. J Ophthalmol 2013;2013:196215.

13 Easty DL, Chignell AH: Fluorescein angiography in anterior segment ischaemia. Br J Ophthalmol 1973;57:18-26.

14 Chen S, Zhou M, Wang W, at al: Levels of angiogenesis-related vascular endothelial growth factor family in neovascular glaucoma eyes. Acta Ophthalmol (Copenh) 2015, Epub ahead of print.
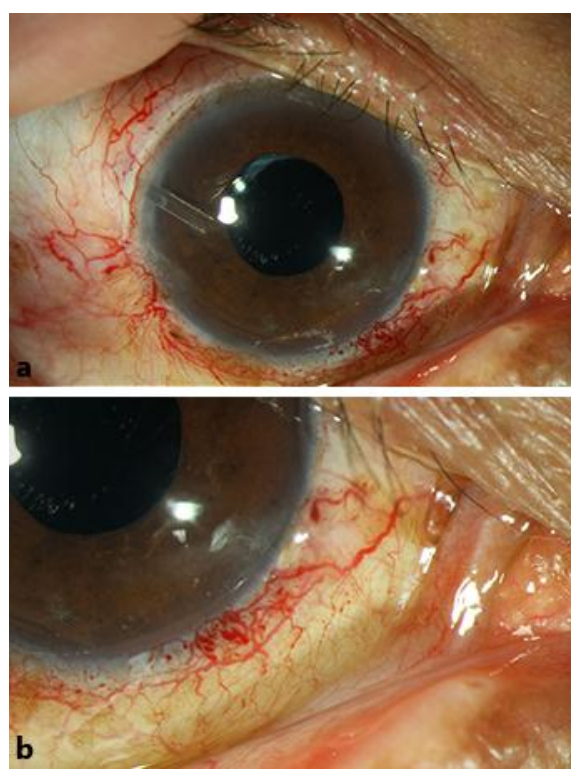

Fig. 1. a Color photographs of the right eye demonstrating the predominance of conjunctival microangiopathy in the inferior bulbar conjunctiva. Pupillary and iris signs are obscured by dilation. $\mathbf{b}$ Microvascular abnormalities: venous dilation, beading, sacculation and tortuosity. Capillary tortuosity, microaneurysms and a reduction in density are shown. Arteriolar thinning results in an abnormal arteriolar/venular ratio. 
Case Reports in

Ophthalmology
Case Rep Ophthalmol 2015;6:272-278

DOI: $10.1159 / 000439266$

(c) 2015 S. Karger AG, Basel

www.karger.com/cop

Abdul-Rahman and Molteno: Conjunctival Microangiopathy in a Patient with Neovascular Glaucoma

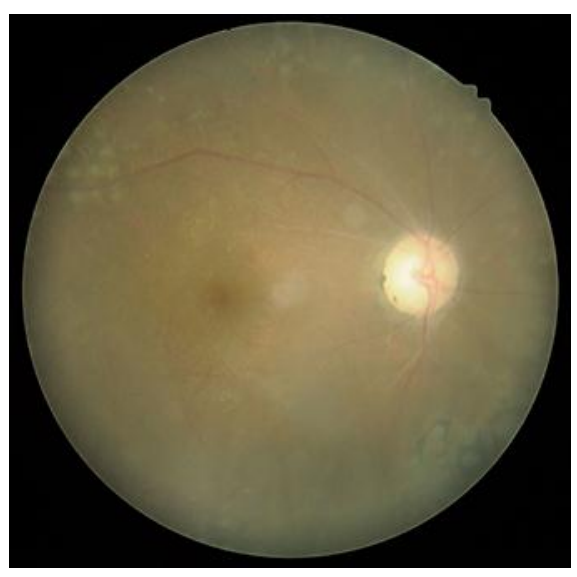

Fig. 2. Color photograph of the posterior segment showing the thin, hyalinized retinal arterioles and peripheral laser scars. There was no evidence of active neovascularization. 
Case Reports in

Ophthalmology

\begin{tabular}{l|l}
\hline \multicolumn{2}{l|}{ Case Rep Ophthalmol 2015;6:272-278 } \\
\hline DOI: $10.1159 / 000439266$ & $\begin{array}{l}\text { C } 2015 \text { S. Karger AG, Basel } \\
\text { www.karger.com/cop }\end{array}$ \\
\hline
\end{tabular}

Abdul-Rahman and Molteno: Conjunctival Microangiopathy in a Patient with Neovascular Glaucoma
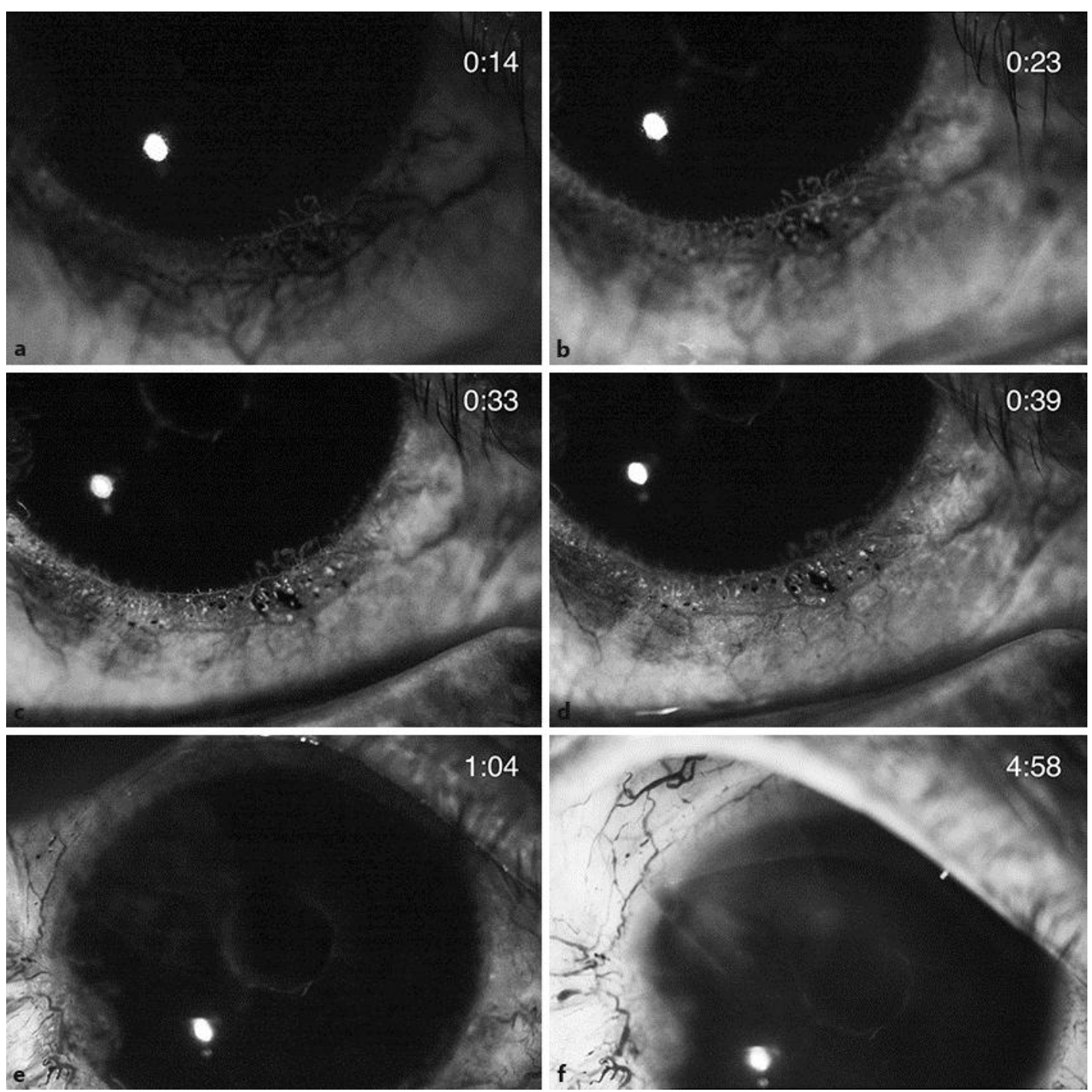

Fig. 3. a-f Anterior segment fluorescein angiography. Time after injection (min:s) is shown in the upper right hand corner. Of note, there is an absence of fluorescein flow in the iris except at the pupillary margin, extensive microaneurysm formation, early arborescent inferonasal corneal neovascularization and a lack of filling in a large saccular dilation at 5 o'clock. e, $f$ Late leakage of the fluorescein dye in the anterior chamber is shown. 